\title{
Benchmarking evolutionary tinkering underlying human-viral molecular mimicry shows multiple host pulmonary-arterial peptides mimicked by SARS-CoV-2
}

\author{
A. J. Venkatakrishnan ${ }^{1}$, Nikhil Kayal ${ }^{1}$, Praveen Anand ${ }^{2}$, Andrew D. Badley $\mathbb{0}^{3}$, George M. Church ${ }^{4}$ and \\ Venky Soundararajan (10
}

\begin{abstract}
The hand of molecular mimicry in shaping SARS-CoV-2 evolution and immune evasion remains to be deciphered. Here, we report 33 distinct 8-mer/9-mer peptides that are identical between SARS-CoV-2 and the human reference proteome. We benchmark this observation against other viral-human 8-mer/9-mer peptide identity, which suggests generally similar extents of molecular mimicry for SARS-CoV-2 and many other human viruses. Interestingly, 20 novel human peptides mimicked by SARS-CoV-2 have not been observed in any previous coronavirus strains (HCOV, SARS-CoV, and MERS). Furthermore, four of the human 8-mer/9-mer peptides mimicked by SARS-CoV-2 map onto HLA-B*40:01, HLA-B*40:02, and HLA-B*35:01 binding peptides from human PAM, ANXA7, PGD, and ALOX5AP proteins. This mimicry of multiple human proteins by SARS-CoV-2 is made salient by single-cell RNA-seq (scRNA-seq) analysis that shows the targeted genes significantly expressed in human lungs and arteries; tissues implicated in COVID-19 pathogenesis. Finally, HLA-A*03 restricted 8-mer peptides are found to be shared broadly by human and coronaviridae helicases in functional hotspots, with potential implications for nucleic acid unwinding upon initial infection. This study presents the first scan of human peptide mimicry by SARS-CoV-2, and via its benchmarking against human-viral mimicry more broadly, presents a computational framework for follow-up studies to assay how evolutionary tinkering may relate to zoonosis and herd immunity.
\end{abstract}

\section{Introduction}

Viral infection typically leads to T-cell stimulation in the host, and autoimmune response associated with viral infection has been observed ${ }^{1}$. SARS-CoV-2, the causative agent of the ongoing COVID-19 pandemic, has complex manifestations ranging from mild symptoms like loss of sense of smell (anosmia) ${ }^{2}$ to severe and critical illness ${ }^{3,4}$. While some molecular factors governing SARS-CoV-2 infection of lung tissues, such as the ACE2 receptor expressing cells have been characterized recently ${ }^{5}$, the

\footnotetext{
Correspondence: Venky Soundararajan (venky@nference.net)

'nference, Cambridge, MA, USA

2nference Labs, Bangalore, India

Full list of author information is available at the end of the article

Edited by I. Amelio
}

mechanistic rationale underlying immune evasion and multi-system inflammation (Kawasaki-like disease) remains poorly understood ${ }^{6,7}$.

The SARS-CoV-2 genome encodes 14 structural proteins (e.g., Spike protein) and nonstructural proteins (e.g., RNA-dependent RNA polymerase), as depicted in Fig. 1a. The nonstructural ORF1ab polyprotein undergoes proteolytic processing to give rise to the following proteins: NSP1, NSP2, PL-PRO, NSP4, 3CL-PRO, NSP6, NSP7, NSP8, NSP9, NSP10, RdRp, Hel, ExoN, NendoU, and 2'O-MT. The human reference proteome consists of 20,350 proteins, which when alternatively spliced, result in over 100,000 protein variants (Fig. 1b) ${ }^{8}$. Here, we investigate the potential for molecular mimicry in the context of immune surveillance and host-antigen recognition in

\section{(c) The Author(s) 2020}

(c) (i) Open Access This article is licensed under a Creative Commons Attribution 4.0 International License, which permits use, sharing, adaptation, distribution and reproduction c. in any medium or format, as long as you give appropriate credit to the original author(s) and the source, provide a link to the Creative Commons license, and indicate if changes were made. The images or other third party material in this article are included in the article's Creative Commons license, unless indicated otherwise in a credit line to the material. If material is not included in the article's Creative Commons license and your intended use is not permitted by statutory regulation or exceeds the permitted use, you will need to obtain permission directly from the copyright holder. To view a copy of this license, visit http://creativecommons.org/licenses/by/4.0/. 
a. n-mer peptide generation

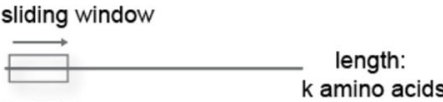

n-mer $\mathrm{n}=8,9 \ldots$

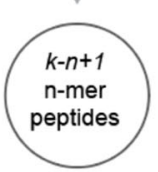

c. Comparison of human-protein mimicking SARS-CoV-2 peptides with peptides from other human coronaviruses
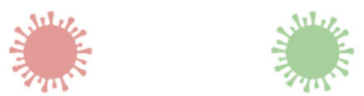

SARS CoV

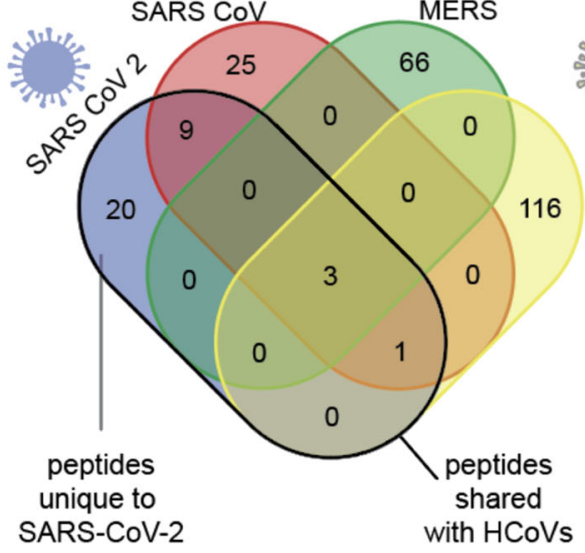

b. Mimicked peptides between SARS-CoV-2 and human proteomes

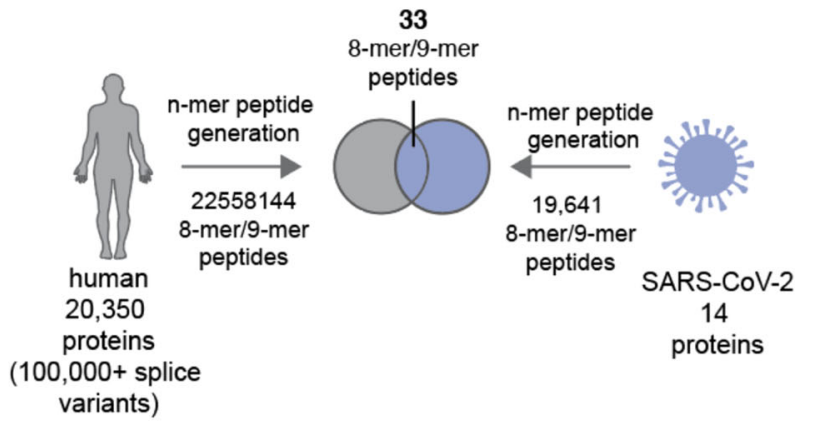

d. Immunomodulatory potential of mimicked peptides from SARS-CoV-2

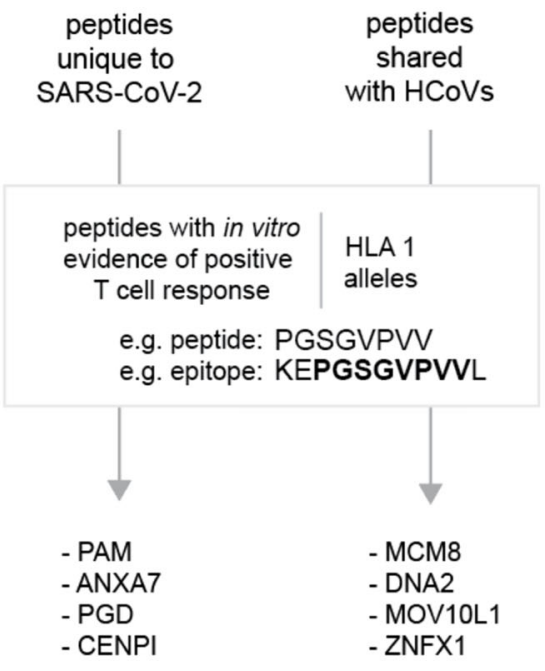

Fig. 1 Molecular mimicry and immunomodulatory potential. a n-mer peptide generation. $\mathbf{b}$ Mimicked peptides between SARS-CoV-2 and human proteomes. c Comparison of human-protein mimicking SARS-CoV-2 peptides with peptides from other human coronaviruses. $\mathbf{d}$ Immunomodulatory potential of mimicked peptides from SARS-CoV-2.

COVID-19, by performing a systematic comparison of known MHC-binding peptides from humans and mapping them onto SARS-CoV-2-derived peptides (see "Methods"). For this, we compute the longest peptides that are identical between SARS-CoV-2 reference proteins and human reference proteins, thus creating a map of COVID-19 host-pathogen molecular mimicry. Based on this resource, we extrapolate the potential for HLA Class-I restricted, T-cell immune stimulation via synthesizing established experimental evidence around each of the mimicked peptides.

\section{Methods}

Computing the SARS-CoV-2 peptides that mimic MHC class-I binding peptides on the human reference proteome

The reference proteome for SARS-CoV-2 consists of 14,221 8-mers and 14,207 9-mers, that result in 9827 distinct 8-mers and 9814 distinct 9-mers (see Table S1). The 8-mers and 9-mers are generated by using a sliding window, moving one amino acid at a time, resulting in overlapping linear peptides. The reference human proteome, on the other hand, consists of 11,211,806 peptides that are 8 -mers and $11,191,459$ peptides that are 9 -mers, resulting in 10,275,893 unique 8 -mers and 10,378,753 unique 9-mers. Including alternatively spliced variants increases the unique peptide counts to $11,215,743$ 8-mers and 11,342,401 9-mers.

Thirty-one 8-mer peptides and two 9-mer peptides are identical between the reference proteomes of SARS-CoV2 and humans, after including alternatively spliced variants (Fig. 1b, Table S2). For comparison, we analyzed the protein sequences from 9434 viral species (taxons) from NCBI RefSeq (see "Methods"). On average, around 45.57 unique 8-mer/9-mers per viral taxon were shared with the 
human proteome $($ mean $=45.57$, median $=12, \quad \mathrm{SD}=$ \pm 135.38 ). In order to control for the complexity and constraints of the amino acid sequences, we also analyzed the distribution of mimicked 8-mers/9-mers normalized by the total number of unique 8 -mers/9-mers present in each viral taxon. On average, a fraction of 0.0028 -mers/9mers out of all the unique 8-mers/9-mers in the virus are identical between the viral proteome and the human proteome (mean $=0.002, \mathrm{SD}= \pm 0.011$ ) (Fig. 1, Supplemental Fig. 1). The fraction of 33 human-mimicking 8mers/9-mers is proximal to the mean. Overall, this suggests that the presence of 33 human-mimicking 8-mers/9mers in SARS-CoV-2 is not surprising compared to the number of human-mimicking 8 -mers/9-mers present in other viruses.

In SARS-CoV-2, no 10-mer or longer peptides are identical between the pathogen and the host reference proteins. Of these, 29 peptides (8-mer/9-mer) mimicked by SARS-CoV-2 map onto nine of the 14 SARS-CoV-2 proteins and 29 of the 20,350 human proteins. By including alternative splicing, the 33 8mers/9-mers mimicked by SARS-CoV-2 map onto 39 of the 100,566 protein splicing variants. That is, $0.16 \%$ of the human proteins and $0.04 \%$ of all the known splicing variants have 8 -mer/9-mer peptides that are mimicked by the SARS-CoV-2 reference proteome. Given that MHC Class I alleles typically engage peptides that are 8-12 mers ${ }^{9}$, the analysis that follows was restricted to mapping host-pathogen mimicry from an immunologic perspective across 8 -mer and 9-mer peptides only.

Comparative analysis of SARS-CoV-2 peptides mimicking the human proteome with the reference SARS, MERS, and seasonal HCoVs

Of the 33 peptides from SARS-CoV-2 that mimic the human reference proteome, 20 peptides are not found in any previous human-infecting coronavirus (SARS, MERS, or seasonal HCoVs) (Table 1). The UniProt database was used to download the 14 protein reference sequences for SARS-CoV. The non-redundant set of protein sequences from other coronavirus strains (HCoV-HKU1:188; HCoV-229E: 246; HCoV-NL63: 330; HCoV-OC43: 910; and MERS: 681 ) was computed by removing $100 \%$ identical sequences, and the remnant sequences were all included in the comparative analysis with SARS-CoV-2 mimicked peptides. A Venn diagram depicting the overlap of mimicked peptides across different human coronaviruses was generated (Fig. 1c).

Given the zoonotic transmission potential of coronaviruses from other organisms to humans, we also considered 8-mer/9-mer peptides derived from 13,431 distinct protein sequences of all non-human coronaviridae from the VIPERdb ${ }^{10}$.

\section{Characterizing the SARS-CoV-2-derived 8-mers/9-mers that mimic established human MHC-binding peptides}

The lengths of the human proteins mimicked by SARS$\mathrm{CoV}-2$ were considered to examine any potential bias towards the larger human proteins (Fig. 1, Supplementary Fig. 1). For instance, human Titin (TTN), despite containing 34,350 amino acids and being of the longest human proteins, does not have even one peptide mimicked by SARS-CoV-2. The longest and shortest human proteins that are mimicked by SARS-CoV-2 are MICAL3 (length $=2002$ amino acids) and BRI3 (length $=$ 125 amino acids), respectively.

The sequence conservation of each mimicked peptide was derived from all the 46,513 sequenced SARS-CoV-2 genomes available in the GISAID database (as on 06/13/ 2020).

The immune epitope database (IEDB) ${ }^{11}$ was used to examine the experimentally established, in vitro evidence for MHC presentation against human or SARS-CoV antigens. The peptides of potential immunologic interest were identified from the IEDB database using the following pair of assays. One of the assays involved purification of specific MHC-class I alleles and estimating the $K_{\mathrm{d}}$ values of specific peptide-MHC complexes through competitive radiolabeled peptide binding ${ }^{12}$. The other assay uses mass spectrometry proteomic profiling of the peptide-MHC complexes, where the MHC complexes were purified from the cell lines specifically engineered to produce mono-allelic MHC class I molecules. The identity of the peptide sequence bound to the class-I MHC molecules was elucidated using mass spectrometry ${ }^{13}$.

\section{Analysis of RNA expression in cells and tissues}

A distribution of RNA expression across all the expressing samples collected from GTEx, Gene expression omnibus, TCGA, and CCLE is created. In this distribution, a high-expression group is defined as the set of samples associated with the top 5\% of expression level. Enrichment score captures the significance of the token in the high-expression group. The significance is captured based on Fisher's test along with BenjaminiHochberg correction. During the comparison of gene expression across tissue types in GTEx, the specificity of expression is computed using "Cohen's D", which is an effect size used to indicate the standardized difference between two means.

\section{Analysis of overlapping peptides between the proteome and viral proteomes}

We analyzed the protein sequences from 9434 viral species (taxons) from NCBI RefSeq (https://ftp.ncbi.nlm. nih.gov/refseq/release/viral/). On average, around 45.57 unique 8-mer/9-mers per viral taxa were detected to be identical to a known human protein $($ mean $=45.57$, 


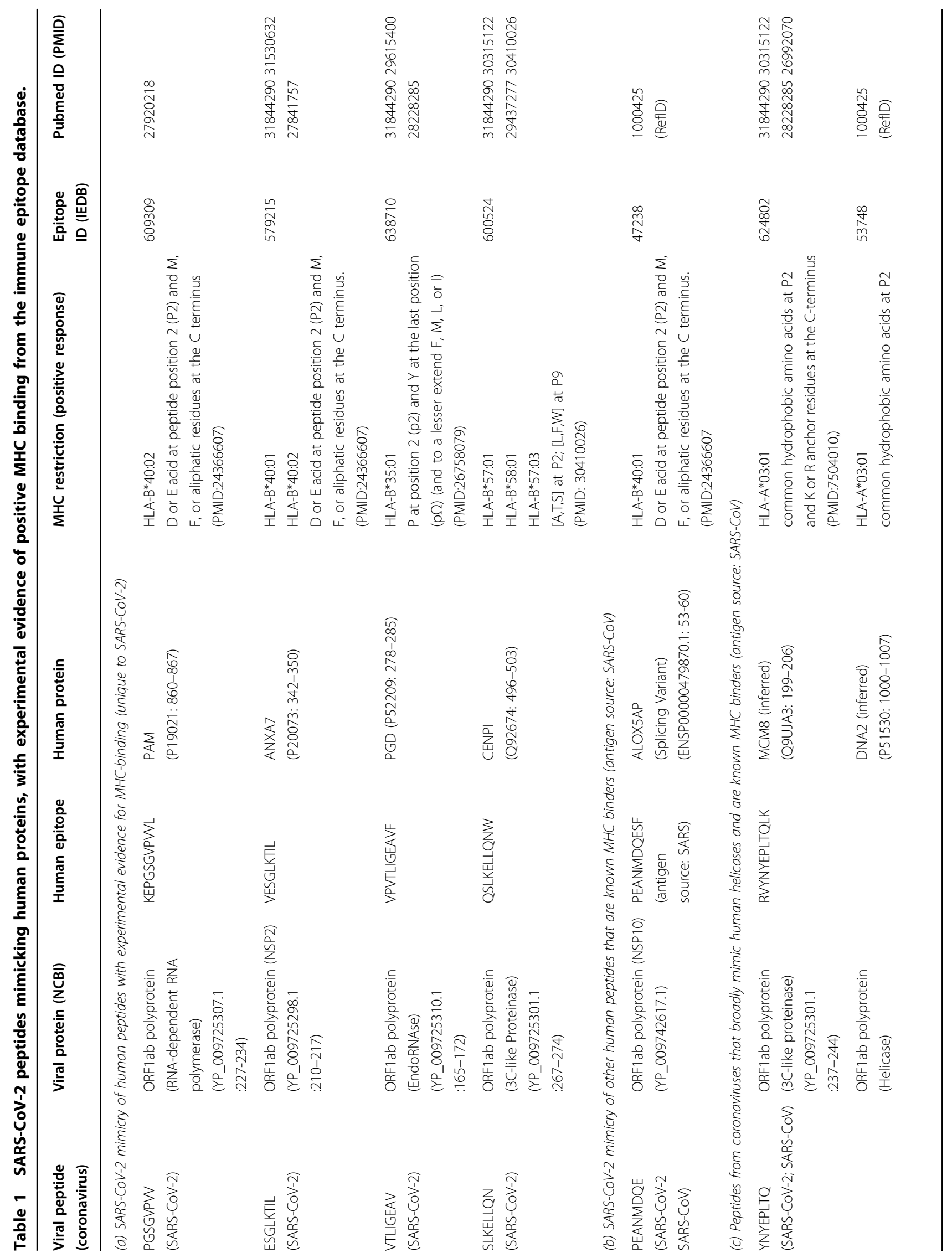




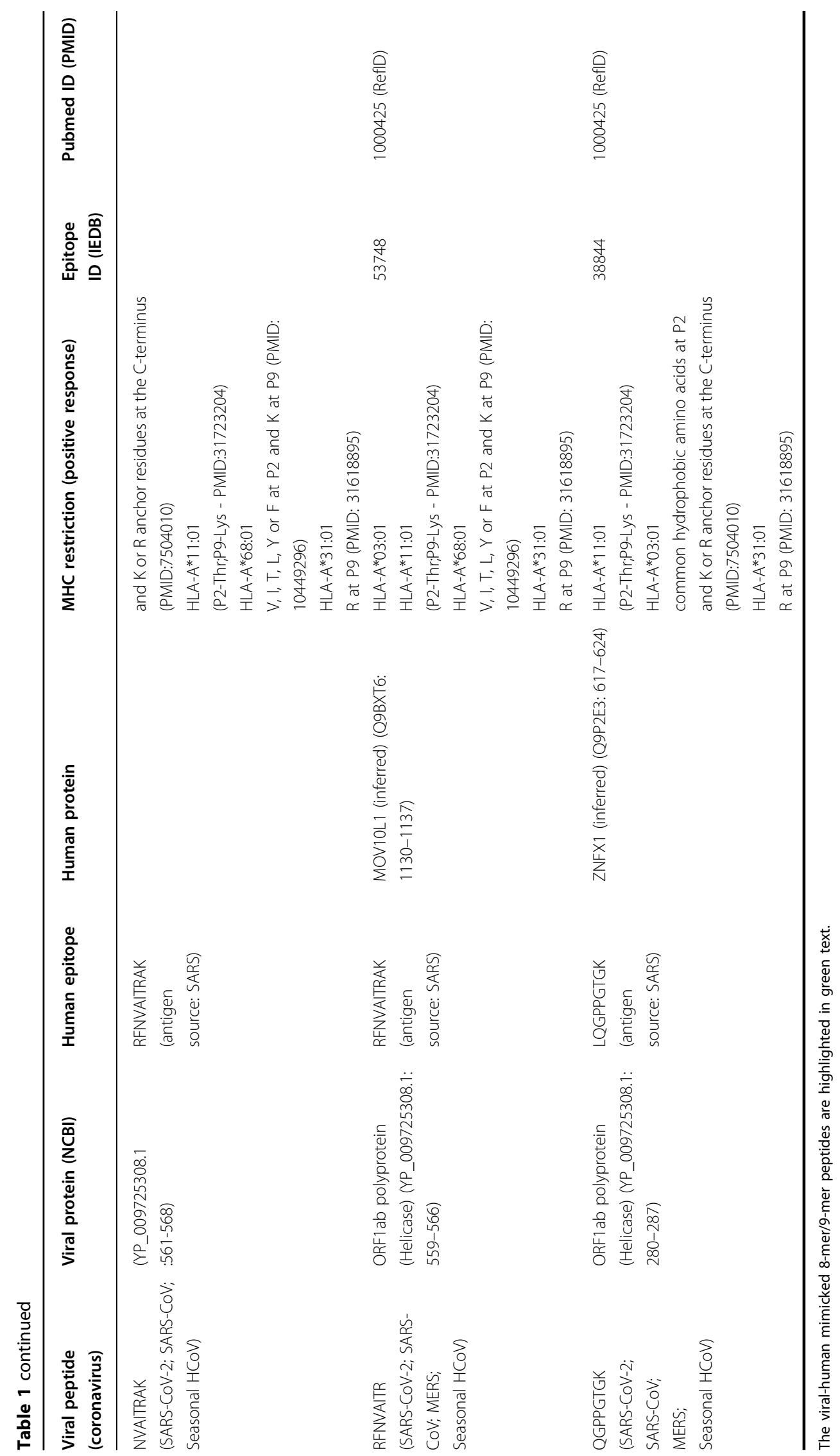


median $=12, \mathrm{SD}= \pm 135.38$ ). The highest number of identical peptides were detected in Pandoravirus dulcis virus with 4423 peptides having an exact identical match to one or more human proteins.

\section{Results \\ Identifying specific human peptides mimicked by SARS- CoV-2 and with in vitro evidence for MHC binding}

A set of 20 8-mer/9-mer human peptides are mimicked by SARS-CoV-2 and no other human coronaviruses (see Methods). Of these 20 peptides, four peptides are constituted within established MHCbinding regions (Table 1-panel a). The four peptides with specific MHC-binding potential that are novel to SARS-CoV-2 map onto the following human proteins: alpha-amidating monooxygenase (PAM), annexin A7 (ANXA7), peptidylglycine 6-phosphogluconate dehydrogenase (PGD), and centromere protein I (CENPI) (Fig. 1d). Analyzing the sequence conservation of the SARS-CoV-2-exclusive peptides shared with the above four human MHC-binding peptides, shows that these SARS-CoV-2 peptides are largely conserved till date (Table 2). The previous human-infecting coronavirus strains (SARS-CoV, MERS, and seasonal $\mathrm{HCoVs}$ ) are notably bereft of these novel SARS-CoV-2 epitopes. An alternatively spliced variant of the human arachidonate 5-lipoxygenase activating protein (ALOX5APENSP00000479870.1; ENST00000617770.4) contains an 8-mer peptide that is mimicked by SARS-CoV-2 as well as SARS-CoV, but not any of the seasonal HCoVs. This peptide has in vitro evidence for positive MHC class-I binding. In addition, there are four human helicases (MCM8, DNA2, MOV10L1, and ZNFX1), each containing peptides with established evidence of MHC classI binding that are also mimicked by SARS-CoV-2 and by previous human-infecting coronaviridae strains (Table 1 -panel c; Fig. 1d) (Table 2).

Novel mimicry of human PAM, ANXA7, and PGD by SARSCoV-2, suggests an enrichment of mimicked peptides in lung, esophagus, arteries, heart, pancreas, and macrophages

Considering bulk RNA-seq data from over 125,000 human samples with non-zero PAM expression shows that PAM is highly expressed in pancreatic islets (enrichment score $=276.9$ across 6 studies and 552 samples), artery (enrichment score $=244.5$ across 3 studies and 343 samples), heart (enrichment score $=$ 243.6 across 19 studies and 442 samples), aorta (enrichment score $=217.1$ across 2 studies and 304 samples), embryonic stem cells (enrichment score $=146.7$ across 2 studies and 352 samples), and fibroblasts (enrichment score $=107.7$ across 28 studies and 215 samples) (Fig. 2a). Among 54 human tissues from
GTEx, PAM is particularly significant within aortic arteries $(n=432$, Cohen's $D=3.1$, mean $=348.2$ TPM $)$ compared to other human tissues. Moderate specificity in gene expression is also noted for the atrial appendage of the heart $(n=429$, Cohen's $D=2.2$, mean $=461.3$ TPM) (Fig. 2b). Further, immunohistochemistry (IHC) based data on 45 human tissues ${ }^{14}$ shows that the PAM protein is detected at high levels in heart muscles, epididymis, and the adrenal gland (Fig. 2b).

Exploring all available human single-cell RNA-seq (scRNA-seq) data shows PAM is expressed in nearly $100 \%$ of pancreatic gamma cells, alpha cells, beta cells, delta cells, and epsilon cells as well as between 50 and $90 \%$ of activated/quiescent stellate cells, acinar cells, endothelial cells, ductal cells of the pancreas. It is also expressed in over $80 \%$ of cardiomyocytes, and $40-70 \%$ of heart fibroblasts, macrophages, endothelial cells, and smooth muscle cells, as well as in $26-27 \%$ of lung pleura fibroblasts, stromal cells, and neutrophils (Fig. 2c). Moreover, analyzing the scRNA-seq data from severe COVID-19 patient's lung bronchoalveolar lavage fluid shows high PAM expression in club cells (Fig. 2d), which intriguingly also express the SARSCoV-2 receptor ACE2 significantly ${ }^{5}$. Furthermore, esophagus scRNA-seq analysis shows esophageal mucosal cells and stromal cells as significant PAM expressors (Fig. 2e). Finally, rarer cell types such as pulmonary neuroendocrine cells and goblet cells of the lungs, and some common cell types like lung serous cells and respiratory secretory cells also express PAM significantly.

Similar to the expression profile of PAM, examining 130,400 human samples with nonzero ANXA7 expression shows that ANXA7 is highly expressed in pancreatic islets (enrichment score $=286.65 ; 543$ samples; 3 studies) and artery (enrichment score $=161.68 ; 184$ samples; 3 studies) (Fig. 3-Supplementary Fig. 1). ANXA7 is significantly expressed in the aortic artery $(n=432$, Cohen's $D=2.1$, mean $=163.1 \mathrm{TPM})$ and the tibial artery $(n=663$, Cohen's $D=2.6$, mean $=176.4$ TPM) (Fig. 3-Supplementary Fig. 2). Analysis of the scRNA-seq data on ANXA7 confirms expression in endothelial cells across multiple tissues and organs, and also indicates expression in lung type- 2 pneumocytes, macrophages, oligodendrocytes (Fig. 3a). Type-2 pneumocytes are noted to express the SARS-CoV-2 receptor ACE2 from scRNA$\mathrm{seq}^{5}$. Analyzing the lung bronchoalveolar lavage fluid scRNA-seq data from patients with severe COVID-19 outcomes shows macrophages, lung epithelial cells, Tcells, club cells, proliferating cells, and plasma cells are significant expressors of ANXA7 (Fig. 3b). Additionally from the study of normal lungs, activated dendritic cells and lymphatic vessel cells are noted to express ANXA7 significantly (Fig. 3c). 


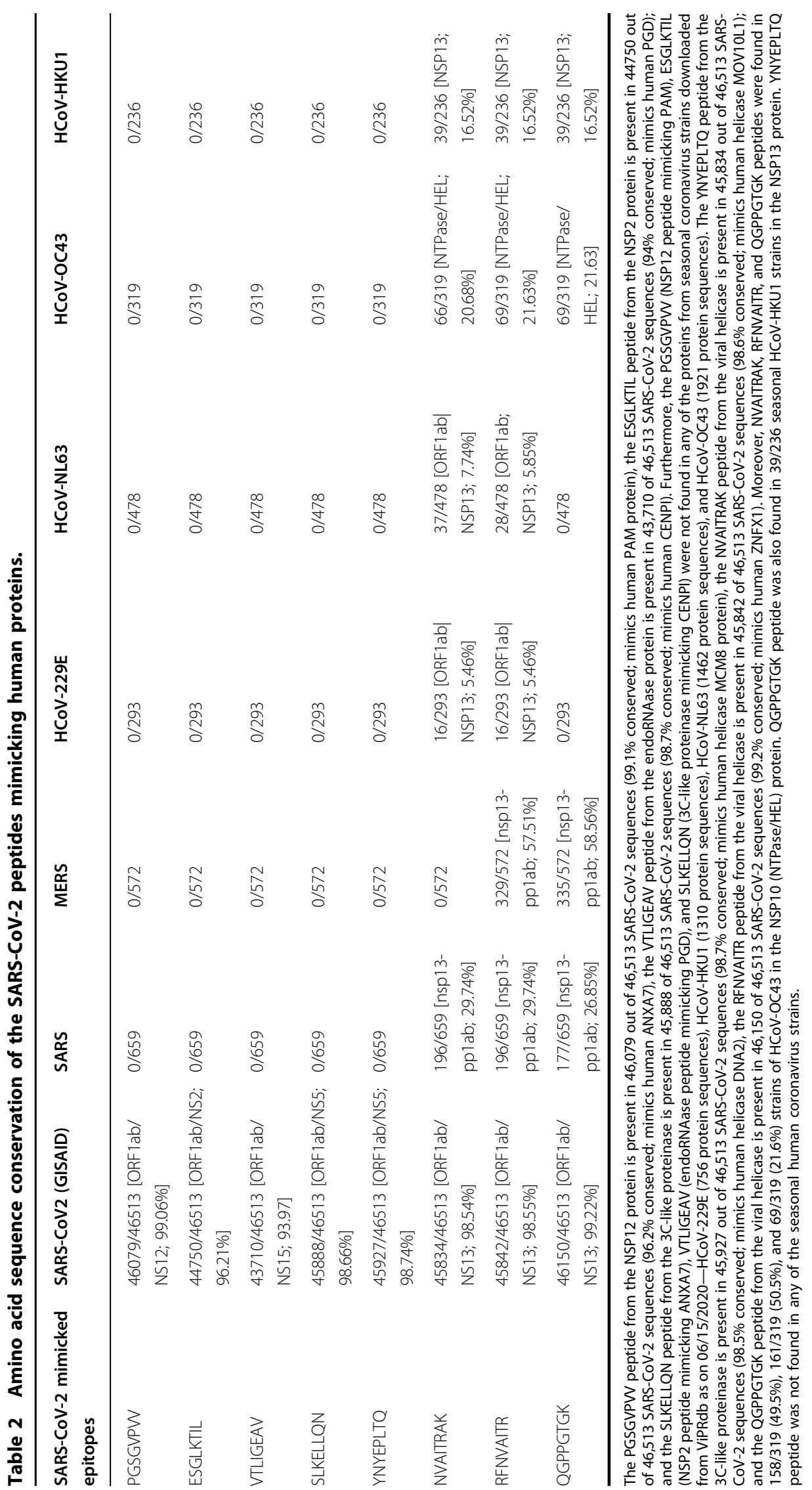



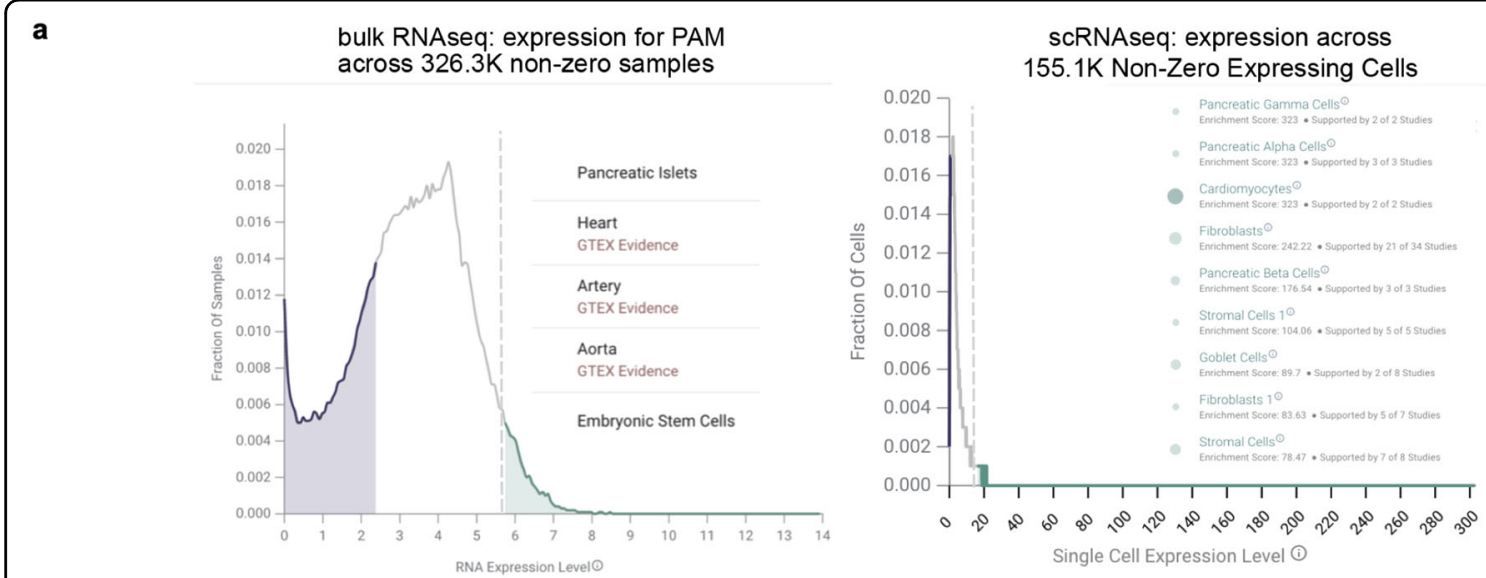

b

nferXRnA Explorer a pam

0 < 뇨 : :

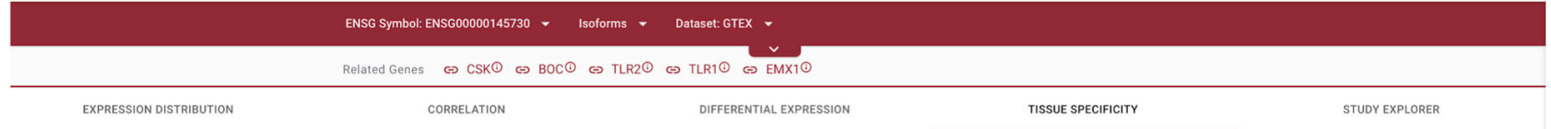

Gene Expression (RNA-Seq)

$$
\text { conselarion }
$$

DIFFERENTIAL EXPRESSION

STUDY EXPLORER

$$
\pm
$$

11. - F. Filter -

Protein expression (immunohistochemistry) $\square$

Tissue

Artery- Aorta
Proten Data

Heart-Atria Anpendobe

Heart- Atrial Appendage
Protten Expression Observed

Artery-Tibial

Artery-Coronary

Areery-Coronary
Proten Data Unavallable

Cervix-Endocervix
Protein Expression Observed

Cells - Cultured Fibroblasts
Protein Data Unavalable

Cervix-Eetocervix

Protein Expression Obsern

Heart - Left Ventricle
Protein Expression Observed

Adrenal Gland

Adrenal Gland
Protelin Expression observed

Pitutary

c

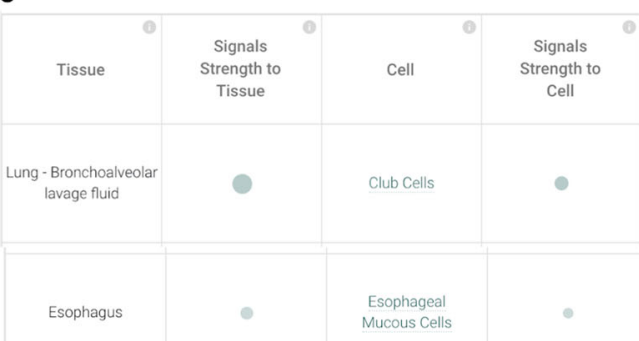

1-10 of 54 > >1 $Q$ t

Healthy Tissu

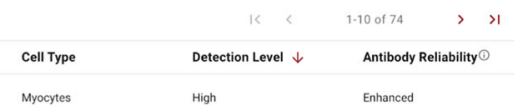

Cohen's
D

Mean (TPM) $\begin{aligned} & \text { Number of } \\ & \text { Samples }\end{aligned}$

Heart Muscle

Myocyes

Enhanced

$3.05 \quad 344.50$

348.21

432

Epididymis

Glandular Cells

Glandular Cells

High

Enhanced

$2.18 \quad 431.00$

$\begin{array}{lll}1.47 & 180.60 & 187.75\end{array}$

$1.12 \quad 162.20$

$1.11 \quad 158.10$

$0.68 \quad 119.35$

$\begin{array}{ll}0.00 & 119.35 \\ 0.63 & 119.80\end{array}$

$0.40 \quad 110.35$

0.12

7893

187.75

$170.67 \quad 240$

Adrenal Gland

Small Intestine Glandular Cells

Pancreas

Glandular Cells

174.50

121.46

124.48

107.72

432

258

${ }_{10}^{240}$

504

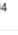

Smooth Muscle

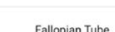

Fallopian Tube

stomach

Nasopharynx

cerebral Cortex

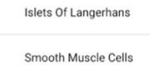

Glandular Cells

\section{Glandular Cells}

Respiratory Epithelial Cells

Neuropil

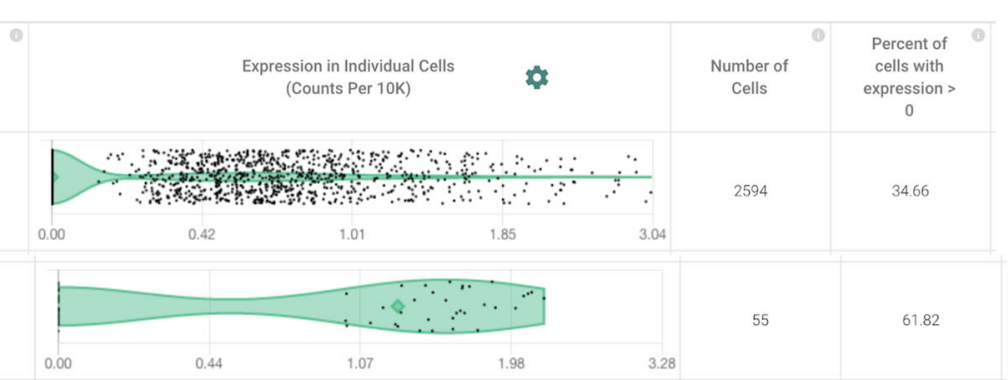

Fig. 2 Multi-omics analysis of human PAM. a (Left) Universal bulk RNA-seq analysis of all available human data shows pancreatic islets, heart, artery, aorta, and embryonic stem cells harbor PAM significantly. (Right) Single-cell RNA-seq (scRNA-seq) confirms high PAM-expressing cells, include multiple pancreatic cells, cardiomyocytes, goblet cells of the lung, bronchus and intestines, stromal cells of the digestive system, and fibroblasts of multiple organs including the lung, trachea, bronchus, intestines, and heart. b Analysis of tissue-specific expression pattern of PAM from bulk RNA-seq (GTEx) and triangulation with IHC antibody staining data (HPA) suggests artery, aorta, and myocytes of the heart muscle as significant PAMexpressing tissues. c Severe COVID-19 patient's lung bronchoalveolar lavage fluid shows high PAM expression in club cells, which also express the SARS-CoV-2 receptor ACE2 (nferX scRNAseq app—lung broncheoalveolar lavage fluid). 


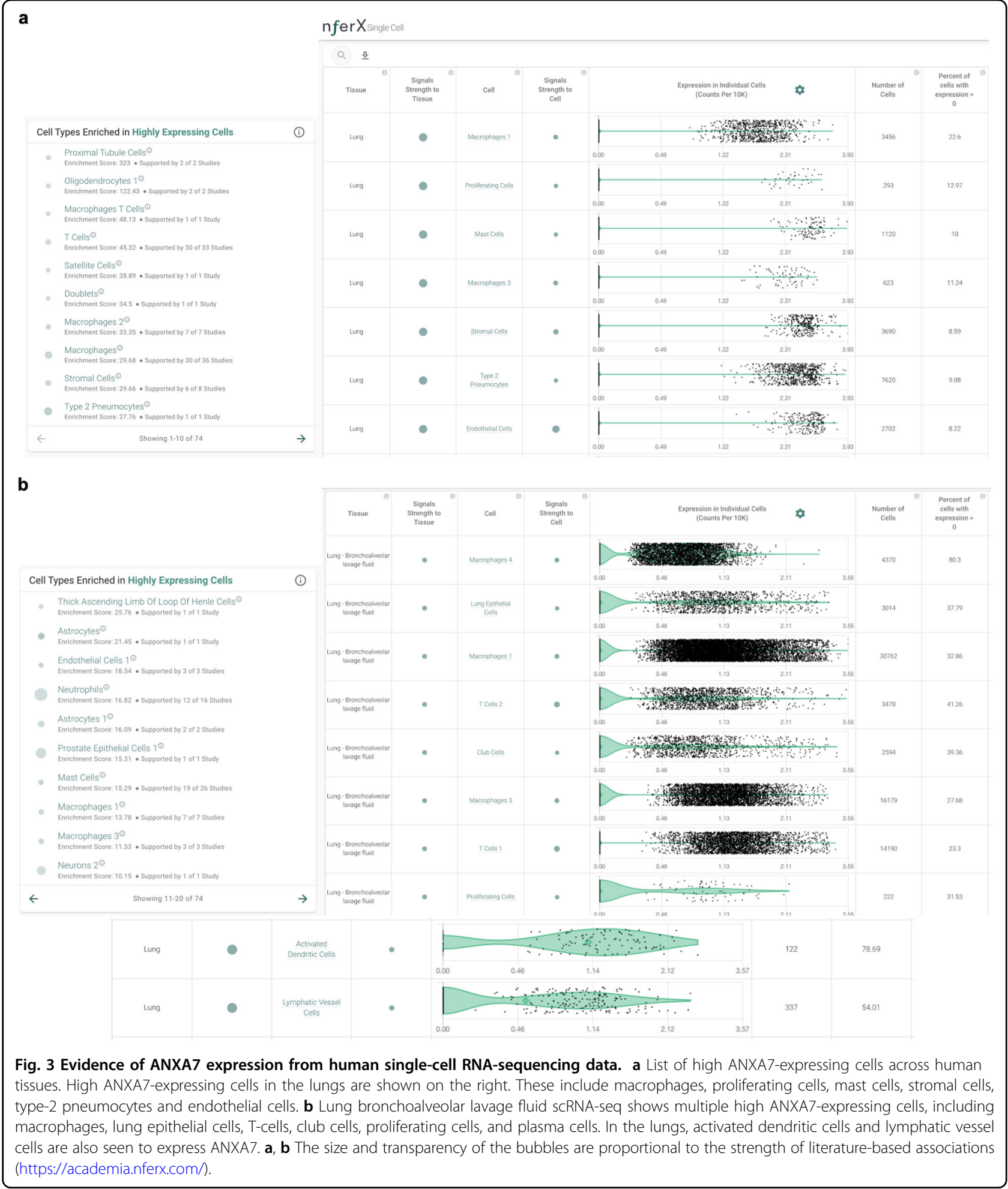

Assessment of around 128,000 human samples shows that PGD is highly expressed in esophagus mucosa (enrichment $=323, n=510,2$ studies), blood (enrichment $=320.5, n=1020$, 39 studies), and macrophages (enrichment $=141.6, n=202$, 4 studies). (Fig. 4, Supplementary
Fig. 1). IHC data on 45 human tissues from the Human Protein Atlas ${ }^{14}$ confirms that PGD is detected at high levels in the esophagus (Fig. 4, Supplementary Fig. 2), and additionally in the testes, tonsils, bone marrow, gallbladder, spleen, and placenta. 


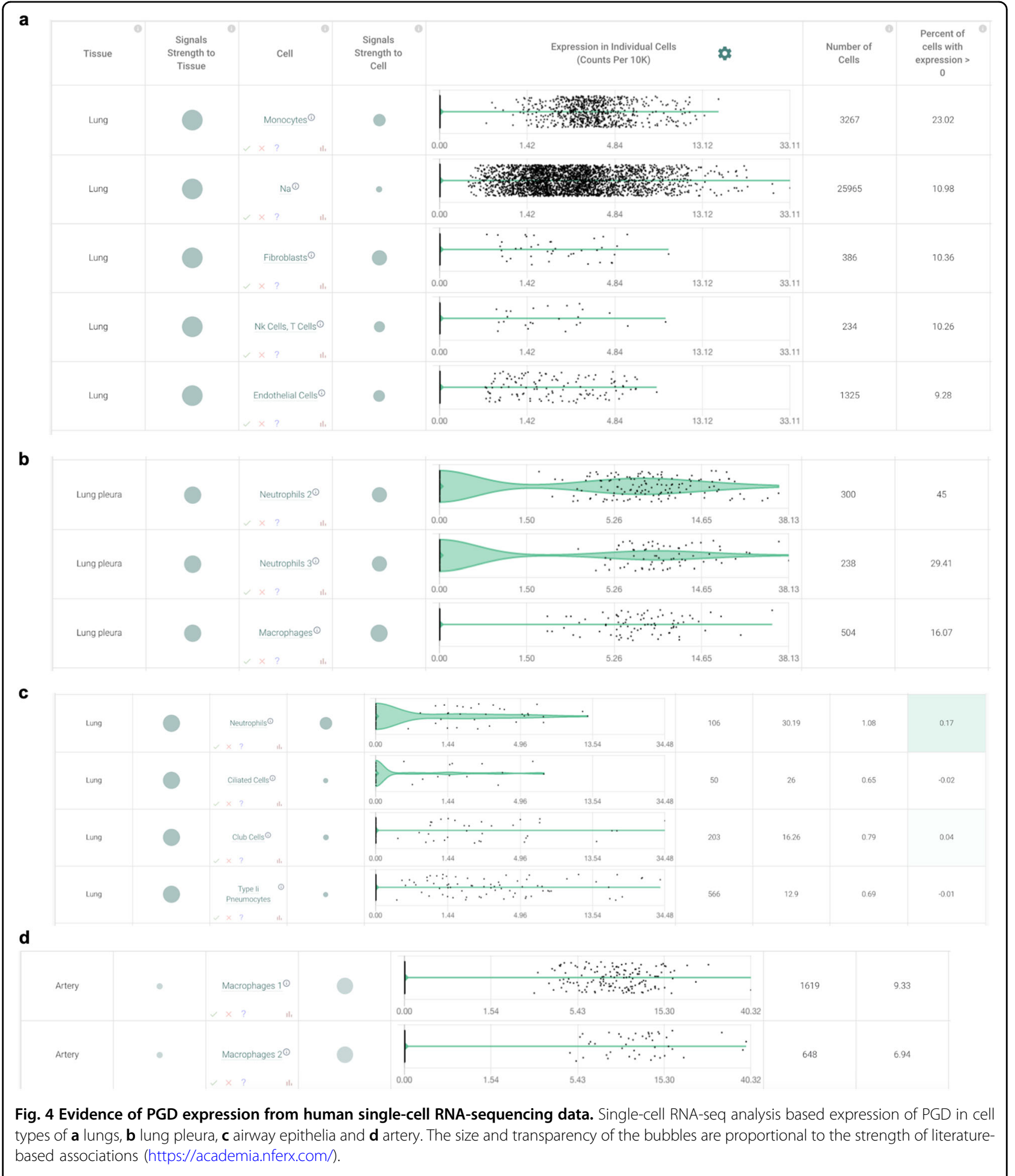

Unlike the expression profiles of PAM, ANXA7, and PGD, CENPI's expression is fairly non-specific and relatively negligible from available data sets. Mild-to-moderate expression of
CENPI is seen in precursor B cells and late erythroid cells, but further studies are needed to ascertain the significance, if any, of CENPI expression, including in the context of COVID-19. 
Multi-pronged mimicry of PAM, ANXA7, and PGD by SARSCoV-2 and its potential for factoring into the pulmonary-arterial autoinflammation seen in severe COVID-19 patients

Positive HLA-B*40:02 binding has been established for the human PAM peptide ("KEPGSGVPVVL") and the ANXA7 peptide ("VESGLKTIL") ${ }^{15-17}$, that contain the distinctive mimicking SARS-CoV-2 peptides (Table 1). The closely related HLA-B*40:01 allele also binds this mimicked ANXA7 peptide ${ }^{17}$. The corresponding mimicking peptides of PAM and ANXA7 are from the viral RNA-dependent RNA polymerase and NSP2 protein, respectively, which are constituted within the MHCbinding regions (highlighted above in bold text). In addition, HLA-B*35:01 has experimental evidence for positive binding of the human PGD peptide mimicked by the SARS-CoV-2 virus (Table 1).

Given the high expression of ANXA7, PGD, and PAM among cells of the respiratory tract, lungs, arteries, cardiovascular system, and pancreas, as well as in macrophages, their striking mimicry by SARS-CoV-2 raises the possibility of individuals with HLA-B*40 and HLA-B*35 alleles being predisposed to potential immune evasion or autoinflammation. Indeed, the potential for broad vascular/ endothelial autoinflammation is consistent with the rarer multi-system inflammatory syndrome or atypical Kawasaki disease noted in few COVID-19-infected children ${ }^{18,19}$.

Alternatively spliced human protein variants analysis for mimicry by SARS-CoV-2 highlights another HLA-B*40:01 restricted protein (ALOX5AP) with autoimmune potential

A splicing variant of ALOX5AP (ENSP00000479870.1 and ENST00000617770.4) containing the 8-mer peptide "PEANMDQE" is one of four alternatively spliced human protein variants that are mimicked by SARS-CoV-2. The other three 8-mer peptides arising from splicing variants do not have any known class I MHC binding reported in the immune epitope database. However, SARS-CoV, which is the only other human-infecting coronavirus in addition to SARS-CoV-2 that also contains PEANMDQE, has been experimentally established to possess the PEANMDQESF epitope that positively binds to the HLAB*40:01 allele.

ALOX5AP is known from literature knowledge synthesis to be associated with ischemic stroke, myocardial infarction, atherosclerosis, cerebral infarction, and coronary artery disease (Fig. 5a). Single-cell RNA-seq studies show numerous types of macrophages expressing ALOX5AP, including in the lungs and brain temporal lobe. Epithelial cells and proliferating cells of the lungs also express ALOX5AP, as do other types of immune cells such as Tcells, neutrophils, and dendritic cells (Fig. 5b). Taken together with the HLA-B*40:01 restricted binding of the PAM and ANXA7 peptides mimicked by SARS-CoV-2, the
ALOX5AP splicing variant also mimicked by SARS-CoV-2 suggests the possibility of immune evasion or autoinflammation in HLA-B*40-constrained COVID-19 patients.

\section{Mimicked HLA-A*03-binding peptides are shared between $\mathrm{HCoV}$ helicases and the human helicases}

There are seven human protein mimicking peptides that are shared between SARS-CoV-2 and at least one other human-infecting coronavirus (Table 1c). These proteins include DNA2, MCM8, MOV10L1, ZNFX1, which are all helicases. Analysis of single-cell RNAseq suggests that the mimicked human proteins are expressed in various celltypes including neuronal cells and immune cells (Fig. 6). The HLA-A*03:01 allele has been established from in vitro experiments to bind SARS-CoV helicase peptides that mimic 8-mer peptides from human MOV10L1, DNA2, ZNFX1, and MCM8 helicases (summarized in Table 1$)^{20}$. The HLA-A*31:01 and HLA-A*11:01 alleles, on the other hand, are known to bind peptides containing the human MOV10L1, DNA2, and ZNFX1 helicase mimics; whereas the HLA-A*68:01 allele has established in vitro evidence of binding peptides containing the human DNA2 and MOV10L1 helicase mimics ${ }^{21}$. In some of the individuals carrying these HLA alleles (Table 1c), a positive T-cell response against their "self" cells that express and display the above coronavirus-mimicked peptides seems plausible.

\section{Discussion}

The presence of identical peptides between viruses and humans has at least two potential implications from an immunologic standpoint. On the one hand, upon presentation of the viral antigens on the surface of infected cells, the virus may evade immune response by masquerading as a host peptide and the recognition of the shared peptides by host regulatory $\mathrm{T}$ cells could promote a generally immunosuppressive environment. On the other hand, an autoimmune response can lead to virus-induced autoinflammatory conditions ${ }^{22}$. Either response requires the coupling of both the presence of the appropriate HLA allele and positive $\mathrm{T}$-cell response toward the mimicked peptide epitopes $^{23}$. It is possible that SARS-CoV-2 leverages one or both of these molecular mimicry strategies to exploit the host immune system. In a small minority of patients who happen to have the unfortunate combination of $\mathrm{MHC}$ restriction and $\mathrm{T}$-cell receptors as mentioned above, the specific tissues and cell types harboring the mimicked protein would bear the brunt of sustained autoimmune damage. The autoimmune lung and vascular damage reported in severe COVID-19 patient mortality ${ }^{24-26}$ necessitates hypothesis-free examination of both these mimicry strategies.

Our study suggests HLA binding of peptides based on existing literature, but existing literature is by no means exhaustive for identifying HLA binding ${ }^{11}$. There is no 


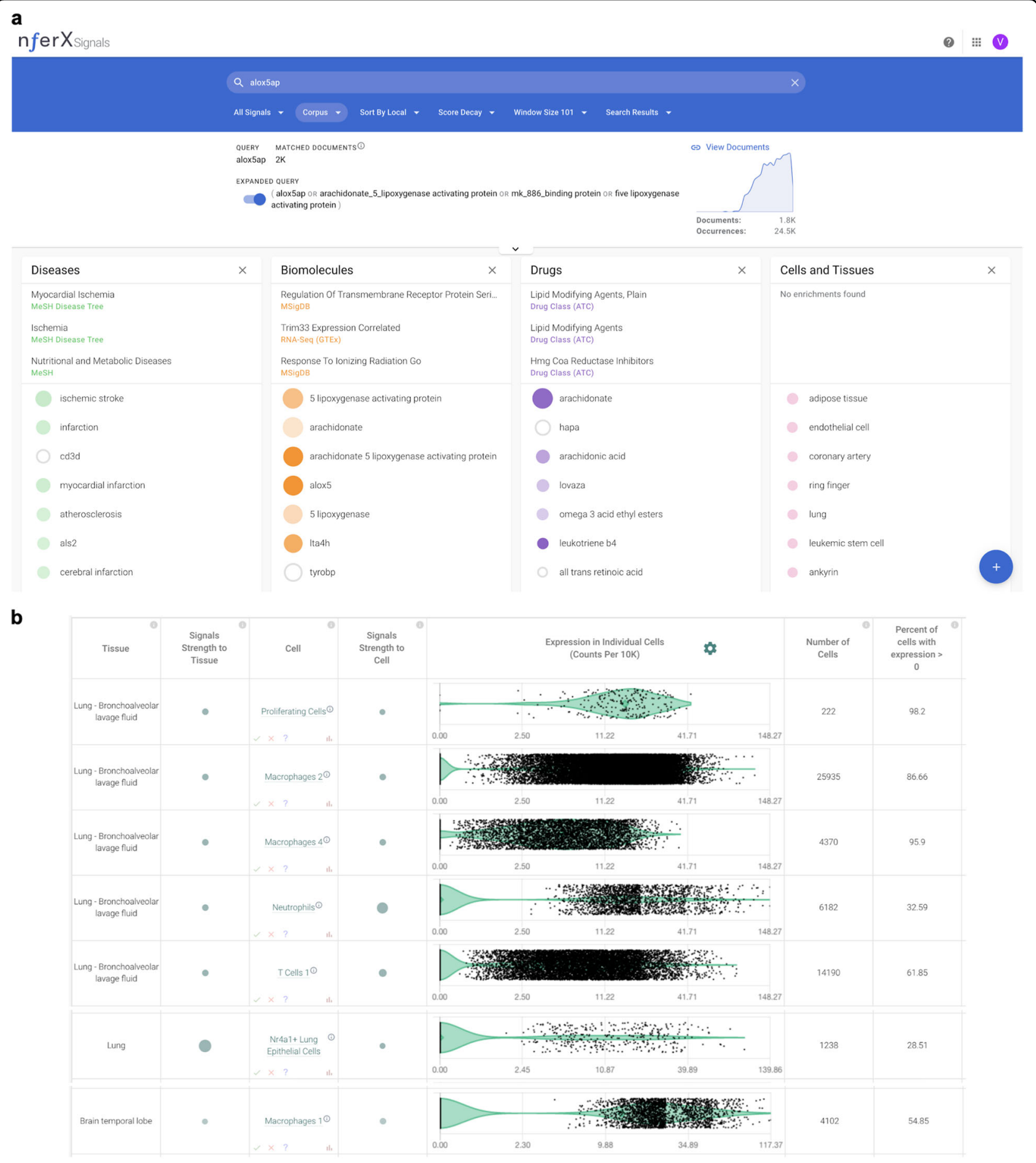

Fig. 5 Evidence for ALOX5AP from biomedical knowledge synthesis and single-cell RNA-seq. a Knowledge synthesis suggests involvement of ALOX5AP in ischemic stroke, myocardial infarction, atherosclerosis, cerebral infarction, and coronary artery disease. b scRNA-seq shows significant expression of ALOX5AP in proliferating cells, macrophages, T-cells, and epithelial cells from the lungs and macrophages of the brain.

known HLA class-I mediated positive T-cell response against certain 8-mers documented in the immune epitope database. For example, GPPGTGKS peptide is shared by the viral helicase and human VPS4A, VPS4B, and SETX.
This peptide is also shared with seasonal human coronaviruses (HCoV-OC43 and HCoV-HKU1) and previous SARS strains (SARS-CoV and MERS). Further experiments are required to assess any potential for autoinflammation. 


\begin{tabular}{|c|c|c|c|}
\hline c. DNA2 & MCM8 & MOV10L1 & ZNFX1 \\
\hline \multicolumn{4}{|l|}{ Glutamatergic $\mathrm{N}$} \\
\hline Granule Neurons $\odot$ & $\begin{array}{l}\text { Rods }^{\circ} \\
\text { Enrichment Score: } 56,15 \text { - Supported by } 1 \text { of } 1 \text { Study }\end{array}$ & $\begin{array}{l}\text { Rods }^{\odot} \\
\text { Enrichment Score: } 52.51 \text { - Supported by } 1 \text { of } 1 \text { Study }\end{array}$ & Neutrophils $^{\odot}$ \\
\hline \multirow{2}{*}{$\begin{array}{l}\text { Granule Neurons } \\
\text { Enrichment Score: } 33.81 \text { - Supported by } 1 \text { of } 1 \text { Study }\end{array}$} & Oligodendrocytes ${ }^{\odot}$ & Proximal Tubule Cells ${ }^{\odot}$ & Enrichment Score: 323 • Supported by 11 of 17 Studies \\
\hline & Enrichment Score: 23.85 - Supported by 1 of 1 Study & Enrichment Score: 44.76 - Supported by 2 of 2 Studies & $\begin{array}{l}\text { Macrophages } 1^{\circ} \\
\text { Enrichment Score: } 23579 \cdot \text { Si }\end{array}$ \\
\hline \multirow{2}{*}{$\begin{array}{l}\text { Gabaergic Neurons } 1{ }^{\odot} \\
\text { Enrichment Score: } 25 \text { - Supported by } 1 \text { of } 1 \text { Study }\end{array}$} & Oligodendrocytes $1^{\odot}$ & Glutamatergic Neurons $1^{\odot}$ & \\
\hline & Enrichment Score: 12.09 S Supported by 2 of 2 Studies & Enrichment Score: 42.4 - Supported by 1 of 1 Study & $\begin{array}{l}\text { Macrophages } 3^{\odot} \\
\text { Enrichment Score: } 104.94 \text { • Supported by } 4 \text { of } 4 \text { Studies }\end{array}$ \\
\hline $\begin{array}{l}\text { Oligodendrocytes } \\
\text { Enrichment Score: } 24.65 \text {. Supported by } 1 \text { of } 1 \text { Study }\end{array}$ & $\begin{array}{l}\text { Glutamatergic Neurons } 1^{\circ} \\
\text { Enrichment Score: } 12.09 \cdot \text { Supported by } 1 \text { of } 1 \text { Study }\end{array}$ & Lung Epithelial Cells ${ }^{\odot}$ & (2) \\
\hline \multirow{2}{*}{$\begin{array}{l}\text { Rods }^{\odot} \\
\text { Enrichment Score: } 20.5 \text { • Supported by } 1 \text { of } 1 \text { Study }\end{array}$} & & ( & Enrichment Score: 82.47 - Supported by 1 of 1 Study \\
\hline & $\begin{array}{l}\text { Neutrophils } 1^{\circ} \\
\text { Enrichment Score: } 11.24 \text { • Supported by } 3 \text { of } 5 \text { Studies }\end{array}$ & $\begin{array}{l}\text { Macrophages } 1^{\odot} \\
\text { Enrichment Score: } 20.79 \text { • Supported by } 4 \text { of } 8 \text { Studies }\end{array}$ & T Cells $^{\odot}$ \\
\hline \multirow{2}{*}{$\begin{array}{l}\text { Late Erythroid Cells }{ }^{\odot} \\
\text { Enrichment Score: } 16.96 \text { - Supported by } 1 \text { of } 1 \text { Study }\end{array}$} & $\mathrm{B}^{\mathrm{C} e l l s}{ }^{\odot}$ & 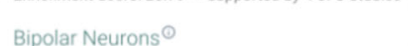 & 1 • Suppor \\
\hline & Enrichment Score: 8.74 - Supported by 12 of 23 Studie & Enrichment Score: 15.64 - Supported by 1 of 1 Study & $\begin{array}{l}\text { Endothelial Cells } 1 \odot \\
\text { Enrichment Score: } 41.94 \text { - Supported by } 3 \text { of } 3 \text { Studies }\end{array}$ \\
\hline $\begin{array}{l}\text { T Cells } 2^{\odot} \\
\text { Enrichment Score: } 9.81 \bullet \text { Supported by } 1 \text { of } 1 \text { Study }\end{array}$ & $\begin{array}{l}\text { Bipolar Neurons }{ }^{\odot} \\
\text { Enrichment Score: } 8.37 \text { - Supported by } 1 \text { of } 1 \text { Study }\end{array}$ & $\begin{array}{l}\text { Gabaergic Neurons } 1^{\odot} \\
\text { Enrichment Score: } 13.85 \text { - Supported by } 1 \text { of } 1 \text { Study }\end{array}$ & $\begin{array}{l}\text { Endothelial Cells }{ }^{\odot} \\
\text { Enrichment Score: } 39.99 \text { - Supported by } 34 \text { of } 45 \text { Studies }\end{array}$ \\
\hline $\begin{array}{l}\text { Gastric Epithelial Cells } 2^{\odot} \\
\text { Enrichment Score: } 7.1 \text { • Supported by } 1 \text { of } 1 \text { Study }\end{array}$ & $\begin{array}{l}\text { Erythroid Cells } 1^{\oplus} \\
\text { Enrichment Score: } 7.62 \text {. Supported by } 2 \text { of } 2 \text { Studies }\end{array}$ & $\begin{array}{l}\text { Theca Cells } \\
\text { Enrichment Score: } 12.07 \text { - Supported by } 1 \text { of } 1 \text { Study }\end{array}$ & $\begin{array}{l}\text { Endothelial Cells } 2^{\circ} \\
\text { Enrichment Score: } 33.3 \text { - Supported by } 3 \text { of } 3 \text { Studies }\end{array}$ \\
\hline $\begin{array}{l}\text { Oligodendrocytes } 1 \odot \\
\text { Enrichment Score: } 7.09 \text { • Supported by } 2 \text { of } 2 \text { Studies }\end{array}$ & $\begin{array}{l}\text { T Cells }{ }^{\odot} \\
\text { Enrichment Score: } 6.86 \text { - Supported by } 25 \text { of } 33 \text { Studie }\end{array}$ & $\begin{array}{l}\text { Granule Neurons } \\
\text { Enrichment Score: } 10.23 \text { - Supported by } 1 \text { of } 1 \text { Study }\end{array}$ & $\begin{array}{l}\text { Glutamatergic Neurons } 1 \odot \\
\text { Enrichment Score: } 24.24 \text { • Supported by } 1 \text { of } 1 \text { Study }\end{array}$ \\
\hline $\begin{array}{l}\text { Astrocytes } 1^{\oplus} \\
\text { Enrichment Score: } 6.56 \cdot \text { Supported by } 1 \text { of } 2 \text { Studies }\end{array}$ & $\begin{array}{l}\text { Granule Neurons }{ }^{\odot} \\
\text { Enrichment Score: } 6.44 \text { - Supported by } 1 \text { of } 1 \text { Study }\end{array}$ & $\begin{array}{l}\text { Muller Glial Cells }{ }^{\odot} \\
\text { Enrichment Score: } 10.16 \text { - Supported by } 1 \text { of } 1 \text { Study }\end{array}$ & $\begin{array}{l}\text { Uterine Epithelial Cells }{ }^{\odot} \\
\text { Enrichment Score: } 21.56 \cdot \text { Supported by } 1 \text { of } 1 \text { Study }\end{array}$ \\
\hline \multicolumn{4}{|c|}{$\begin{array}{l}\text { Fig. } 6 \text { Expression of mimicked human helicases. scRNAseq based expression analysis of human helicases containing peptides mimicked by } \\
\text { helicases in SARS-CoV-2 and other human coronaviruses (https://academia.nferx.com/). }\end{array}$} \\
\hline
\end{tabular}

Although our current study focussed on humaninfecting coronaviruses, molecular mimicry is expected to exist beyond human-infecting coronaviruses. A stringent BLAST search was also performed for all the four immunomodulatory peptides specific to SARS-CoV-2 against all the sequences of Coronaviridae family in the nonredundant protein database. There were no hits found outside the orthocoronavirinae family for these peptides. An exact match for peptides-"PGSGVPVV", "VTLIGEAV", and "SLKELLQN" was found only in either the pangolin coronavirus or the Bat coronavirus RaTG13. An exact match for "PGSGVPVV" was also found in Canada goose coronavirus (YP_009755895.1). The human ANXA7-mimicking peptide "ESGLKTIL" is, however, noted only in SARS-CoV-2 sequences, with the closest known evolutionary homologs attributed to BAT SARSlike coronavirus (ESGLKTIL), the NL63-related bat coronavirus strains, and the recently sequenced pangolin coronavirus (Fig. 3, Supplementary Fig. 2). Our observed multi-pronged human mimicry of distinct SARS-CoV-2 peptides may owe their origins to zoonotic transmission from coronaviruses circulating within pangolins and bats as natural reservoirs, aided by genetic recombination and purifying selection ${ }^{27,28}$. Our hypothesis-free computational analysis of all available sequencing data, from genomic sequencing and single-cell transcriptomics, sets the stage for targeted experimental interrogation of immuno-evasive or immuno-stimulatory roles of the mimicked peptides within zoonotic reservoirs and human subjects alike. Such a holistic data sciences-enabled "wet lab" platform for characterizing molecular mimicry and its immunologic implications may help shine a new light on the relentless evolutionary tinkering that propels the rise and fall of viral pandemics.

\section{Acknowledgements}

The authors thank Patrick Lenehan, Travis Hughes, and Murali Aravamudan for their thoughtful feedback.

\section{Author details}

${ }^{1}$ nference, Cambridge, MA, USA. ${ }^{2}$ nference Labs, Bangalore, India. ${ }^{3}$ Department of Infectious Diseases, Mayo Clinic, Rochester, MN, USA. ${ }^{4}$ Department of Genetics, Harvard Medical School, Boston, MA, USA

\section{Conflict of interest}

The authors declare that they have no conflict of interest.

\section{Publisher's note}

Springer Nature remains neutral with regard to jurisdictional claims in published maps and institutional affiliations.

The online version of this article (https://doi.org/10.1038/s41420-020-00321-y) contains supplementary material, which is available to authorized users.

Received: 18 August 2020 Accepted: 1 September 2020

Published online: 02 October 2020

\section{References}

1. Smatti, M. K. et al. Viruses and Autoimmunity: A Review on the Potential Interaction and Molecular Mechanisms. Viruses 11, 762. https://doi.org/ 10.3390/v11080762 (2019).

2. Sayin, I., Yaşar, K. K. \& Yazici, Z. M. Taste and Smell Impairment in COVID-19: An AAO-HNS Anosmia Reporting Tool-Based Comparative Study. Otolaryngol Head Neck Surg 2020 163, 473-479, https://doi.org/ 10.1177/0194599820931820 (2020).

3. Chen, T. et al. Clinical characteristics of 113 deceased patients with coronavirus disease 2019: retrospective study. Br. Med. J. 368, m1091 (2020). 
4. Wagner, T. et al. Augmented curation of clinical notes from a massive EHR system reveals symptoms of impending COVID-19 diagnosis. elife $\mathbf{9}$, e58227. https://doi.org/10.7554/eLife.58227 (2020).

5. Venkatakrishnan, A. et al. Knowledge synthesis of 100 million biomedica documents augments the deep expression profiling of coronavirus receptors. elife 9, e58040. https://doi.org/10.7554/eLife.58040 (2020).

6. Verdoni, L. et al. An outbreak of severe Kawasaki-like disease at the Italian epicentre of the SARS-CoV-2 epidemic: an observational cohort study. Lancet 395, 1771-1778 (2020).

7. Caso, F. et al. Could Sars-coronavirus-2 trigger autoimmune and/or autoinflammatory mechanisms in genetically predisposed subjects? Autoimmun. Rev. 19, 102524 (2020)

8. UniProt Consortium. UniProt: a worldwide hub of protein knowledge. Nucleic Acids Res 47, D506-D515. https://academic.oup.com/nar/article/47/D1/D506/ 5160987 (2019).

9. Trolle, T. et al. The length distribution of class I-restricted T cell epitopes is determined by both peptide supply and MHC allele-specific binding preference. J. Immunol. 196, 1480-1487 (2016).

10. Carrillo-Tripp, M. et al. VIPERdb2: an enhanced and web API enabled relational database for structural virology. Nucleic Acids Res. 37, D436-D442 (2009).

11. Fleri, W. et al. The immune epitope database and analysis resource in epitope discovery and synthetic vaccine design. Front. Immunol. 8, 278 (2017).

12. Sidney, J. et al. Measurement of $\mathrm{MHC} /$ peptide interactions by gel filtration or monoclonal antibody capture. Curr. Protoc. Immunol. Chapter 18, Unit 18.3 (2013).

13. Abelin, J. G. et al. Mass spectrometry profiling of HLA-associated peptidomes in mono-allelic cells enables more accurate epitope prediction. Immunity 46, 315-326 (2017).

14. Uhlén, M. et al. Proteomics. Tissue-based map of the human proteome. Science 347, 1260419 (2015).

15. Ramarathinam, S. H. et al. Identification of native and posttranslationally modified HLA-B*57:01-restricted HIV envelope derived epitopes using immunoproteomics. Proteomics 18, e1700253 (2018).
16. Lorente, E. et al. Substantial influence of ERAP2 on the HLA-B*40:02 peptidome: implications for HLA-B*27-negative ankylosing spondylitis. Mol. Cell. Proteom. 18, 2298-2309 (2019).

17. Pearson, $\mathrm{H}$. et al. MHC class I-associated peptides derive from selective regions of the human genome. J. Clin. Investig. 126, 4690-4701 (2016).

18. Belhadjer, Z. et al. Acute heart failure in multisystem inflammatory syndrome in children (MIS-C) in the context of global SARS-CoV-2 pandemic. Circulation https://doi.org/10.1161/CIRCULATIONAHA.120.048360 (2020).

19. Viner, R. M. \& Whittaker, E. Kawasaki-like disease: emerging complication during the COVID-19 pandemic. Lancet 395, 1741-1743 (2020).

20. Vita, R. et al. The Immune Epitope Database (IEDB): 2018 update. Nucleic Acids Res. 47, D339-D343 (2019).

21. Vita, R. et al. The immune epitope database (IEDB) 3.0. Nucleic Acids Res. 43 D405-D412 (2015).

22. Getts, D. R., Chastain, E. M. L., Terry, R. L. \& Miller, S. D. Virus infection, antiviral immunity, and autoimmunity. Immunol. Rev. 255, 197-209 (2013).

23. Fujinami, R. S., von Herrath, M. G., Christen, U. \& Whitton, J. L. Molecular mimicry, bystander activation, or viral persistence: infections and autoimmune disease. Clin. Microbiol. Rev. 19, 80-94 (2006).

24. Carsana, L. et al. Pulmonary post-mortem findings in a series of COVID-19 cases from northern Italy: a two-centre descriptive study. Lancet Infect. Dis. https://doi.org/10.1016/\$1473-3099(20)30434-5 (2020).

25. Varga, Z. et al. Endothelial cell infection and endotheliitis in COVID-19. Lancet 395, 1417-1418 (2020).

26. Ackermann, M. et al. Pulmonary vascular endothelialitis, thrombosis, and angiogenesis in Covid-19. N. Engl. J. Med. https://doi.org/10.1056/ NEJMoa2015432 (2020).

27. Li, X. et al. Emergence of SARS-CoV-2 through recombination and strong purifying selection. Sci. Adv. eabb9153 (2020).

28. Zhang, T., Wu, Q. \& Zhang, Z. Probable pangolin origin of SARS-CoV-2 associated with the COVID-19 outbreak. Curr. Biol. 30, 1346-1351.e2 (2020). 